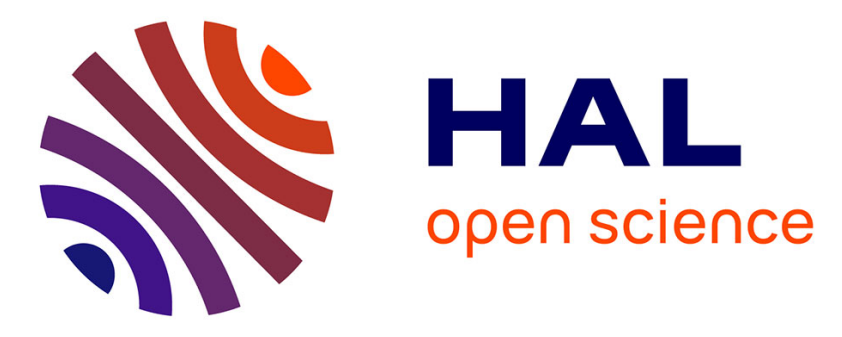

\title{
Association entre hidrosadénite suppurative et spondyloarthrite
}

Marion Fauconier, Ziad Reguiai, Coralie Barbe, Alban Colosio, Jean-Paul

Eschard, Jean-Hugues Salmon, Guillaume Direz

\section{To cite this version:}

Marion Fauconier, Ziad Reguiai, Coralie Barbe, Alban Colosio, Jean-Paul Eschard, et al.. Association entre hidrosadénite suppurative et spondyloarthrite. Revue du Rhumatisme, 2019, 86, pp.283 - 287. 10.1016/j.rhum.2019.03.001 . hal-03485088

\section{HAL Id: hal-03485088 \\ https://hal.science/hal-03485088}

Submitted on 20 Dec 2021

HAL is a multi-disciplinary open access archive for the deposit and dissemination of scientific research documents, whether they are published or not. The documents may come from teaching and research institutions in France or abroad, or from public or private research centers.
L'archive ouverte pluridisciplinaire HAL, est destinée au dépôt et à la diffusion de documents scientifiques de niveau recherche, publiés ou non, émanant des établissements d'enseignement et de recherche français ou étrangers, des laboratoires publics ou privés.

\section{다)(1) $(5$}

Distributed under a Creative Commons Attribution - NonCommercial| 4.0 International 


\section{Association entre hidrosadénite suppurative et spondyloarthrite}

Marion Fauconier ${ }^{1}$, Ziad Reguiai ${ }^{2}$, Coralie Barbe ${ }^{3,4}$, Alban Colosio ${ }^{5}$, Jean-Paul Eschard ${ }^{1}$, Jean-Hugues Salmon ${ }^{1,4}$, Guillaume Direz ${ }^{1,6}$

1 CHU Reims, Hôpital Maison Blanche, Service de Rhumatologie, 51092, France

2 CHU Reims, Hôpital Robert Debré, Service de Dermatologie, 51092, France

3 CHU Reims, Hôpital Robert Debré, Unité d'Aide Méthodologique, 51092, France

4 Université de Reims Champagne Ardenne, Faculté de Médecine, EA 3797, REIMS, 51095, France

5 CHU Reims, Hôpital Robert Debré, Service de Radiologie, 51092, France

$6 \mathrm{CH}$ Le Mans, Service de rhumatologie, 72037, France

\section{Correspondance :}

Marion Fauconier, service de Rhumatologie, Hôpital Maison Blanche, CHU REIMS 45 rue cognac jay 51092 Reims Cedex Tel : +33 326784462 Fax : +33 3267845 50, mail mfauconier@chu-reims.fr 


\section{Abstract}

Objectifs : Nous avons souhaité enquêter sur le lien entre hidrosadénite suppurative (HS) et spondyloarthrite (SpA).

Méthodes: Nous avons effectué une étude transversale monocentrique chez des patients atteints de HS, suivis dans un centre de soins tertiaire, et chez des volontaires sans affection dermatologique, ayant le même âge ( \pm 5 ans) et le même sexe. Pour chaque sujet inclus, examen clinique, recherche de HLA-B27 et IRM sacro-iliaque ont été effectués afin de diagnostiquer la SpA selon les critères ASAS.

Résultats : Au total, 39 sujets ont été testés dans chaque groupe $(70 \%$ de femmes dans chaque groupe ; âge moyen $35,6 \pm 11,1$ ans pour le groupe exposé et $36,0 \pm 11,1$ ans pour le groupe témoin). Onze patients (28,2\%) ayant un HS présentaient une SpA (diagnostic déjà connu pour 4 d'entre eux). La spondyloarthrite était aussi présente chez un sujet sain (2\%) (RC 11,0;95\% IC 4,1-83,3; $p=0,02$ ). La spondylarthrite axiale, forme la plus courante, a été observée chez 9 patients sur 11 , chez les autres il s'agissait de spondyloarthrite périphérique. Dans le groupe HS seul le HLA B27 a été trouvé comme facteur associé.

Conclusions: Nos résultats indiquent que l'hidrosadénite suppurative est significativement associé à un risque accru de spondyloarthrite, indépendamment de l'âge et du sexe. Les patients atteints d'hidrosadénite suppurative présentant des symptômes ostéoarticulaires, particulièrement les douleurs lombaires ou la dactylite, devraient faire l'objet d'une surveillance pour déceler la spondylarthrite.

Mots clés : spondyloarthrite ; hidrosadénite suppurative ; inflammation ; imagerie par résonance magnétique. 


\section{Introduction}

L'hidrosadénite suppurative (HS) est une maladie de peau chronique se manifestant par une inflammation de l'épithélium folliculaire des glandes apocrines [1]. Elle évolue vers des écoulements de pus, des fistules et une cicatrisation en relief [2] II s'agit d'une pathologie extrêmement invalidante, nuisant fortement à la qualité de vie des patients [3]. Elle affecte 1 à $4 \%$ de la population [4], avec une prédominance féminine (sex-ratio 4/1) [5,6]. Cette maladie reste méconnue, et le retard diagnostique est en moyenne de 7 ans [7] Ce délai est lié à une mauvaise connaissance par le corps médical de la maladie, pourtant le diagnostic de cette affection dermatologique est clinique, et ne nécessite pas d'examen complémentaire L'HS est une pathologie inflammatoire, qui se complique d'une surinfection bactérienne secondaire résultant de l'obstruction folliculaire [8] En effet il a été mis en évidence chez des patients atteints d'HS des taux sériques élevés de Tumor Necrosis Factor $\alpha$ (TNF $\alpha$ ) proches de ceux rencontrés dans les rhumatismes inflammatoires [9]. Les taux de TNF $\alpha$, interleukine (IL) IL-1 $\beta$ et IL 10 sont également augmentés sur les biopsies cutanées [10]. Récemment, l'adalimumab, un inhibiteur du TNF $\alpha$ vient d'obtenir une AMM dans les formes sévères de cette pathologie.

L'hypothèse d'un lien entre HS et rhumatisme inflammatoire est ancienne, étayée par plusieurs cas cliniques ou de courtes séries [11-13]. Le rhumatisme inflammatoire le plus fréquemment rapporté était la spondyloarthrite ( $\mathrm{SpA})$. Plus récemment, une étude prospective multicentrique a démontré une prévalence plus élevée de SpA dans une population HS par rapport à celle de la population générale [14].

L'ensemble de ces éléments nous fait penser qu'il existe un lien entre ces maladies. Cependant, les données de la littérature sont rares. II s'agit d'études observationnelles descriptives, anciennes et rétrospectives ; elles n'utilisent pas les méthodes diagnostiques récentes comme l'IRM et les nouvelles classifications des SpA.

Jusqu'à présent, aucune étude observationnelle analytique n'a étudié ou démontré de lien entre ces deux pathologies. 
Nous rapportons ici les résultats de notre étude dont l'objectif principal était de tester I'hypothèse d'un risque de SpA plus élevé chez les patients suivis pour HS Nous avons également étudié les caractéristiques de ces SpA et recherché s'il existait des facteurs associés.

\section{Méthodes}

\subsection{Design de l'étude}

II s'agissait d'une étude transversale de type exposés/non-exposés, monocentrique s'intégrant dans le cadre d'un projet hospitalier de recherche clinique.

Cette étude a été approuvée par le comité éthique de Dijon Est I (№2012-A0084142), et par l'agence nationale de sécurité du médicament et des produits de santé (ANSM). Tous les patients ont signé un formulaire de consentement éclairé.

\subsection{Inclusions}

Les patients exposés ont été extraits à partir de la base informatique ; il s'agissait de patients suivis pour HS dans le service de dermatologie du CHU de Reims. Nous avons inclus les patients quel que soit le stade de sévérité de la maladie ou la durée d'évolution de cette dernière.

Les patients non-exposés étaient des sujets non HS, appariés aux sujets exposés sur l'âge $( \pm 5$ ans) et le sexe. Les patients chez qui une spondyloarthrite était déjà connue n'ont pas été exclus de l'étude. Les patients non exposés ont été recrutés via un affichage sur le portail intranet de l'hôpital, mais également au centre de promotion de santé de la Marne.

\subsection{Data recorded}

Pour les patients exposés, la sévérité de HS a été évaluée par :

- le grade de Hurley $[15,16]$

1 : abcès unique ou multiple sans fistule, ni processus cicatriciel fibreux.

2: abcès récidivants avec formation de fistules et de cicatrices hypertrophiques. Lésion(s) uniques ou multiples séparées les unes des autres. 
3 : atteinte diffuse ou quasi diffuse, ou fistules interconnectées et abcès sur toute l'étendue de la zone d'atteinte.

Le grade de Hurley le plus élevé au cours de la maladie était retenu et non celui à l'inclusion.

- l'index dermatologique de qualité de vie (index DLQI) évaluant le retentissement de HS sur la vie quotidienne des patients.

Pour tous les patients inclus, le diagnostic de SpA était posé par un rhumatologue (GD) selon les critères ASAS (Assessement of SpondyloArthritis Society)[17,18] [18]Les patients analysés dans cette étude ont tous bénéficié d'un examen clinique complet afin de poser le diagnostic de lombalgie inflammatoire, une recherche de l'antigène HLA B27, un dosage de la CRP et une IRM des sacro-iliaques.

\subsection{Protocole IRM}

Les IRM des sacro-iliaques ont été réalisées sur une IRM 1,5 Tesla avec des coupes axiales et coronales obliques en séquences T1 et T2 STIR (Short T Inversion Recovery), et ont été interprétées par un rhumatologue (GD) et un radiologue expérimentés $(A C)$ en aveugle et de manière indépendante. Les analyses discordantes ont été revues par les 2 lecteurs ensemble pour obtenir un consensus. Le lecteur de l'IRM statuait quant à l'existence ou non une d'une sacro-iliite inflammatoire répondant aux critères de l'ASAS [19] et/ou sacro-iliite structurale (en cas de sclérose étendue associée à au moins 2 érosions ou une ankylose) [20]

\subsection{Type de spondyloarthrite}

Les $\mathrm{SpA}$ ont été classées en $\mathrm{SpA}$ axiales et périphériques selon les critères définis en 2009 par l'ASAS [18].

Dans cette étude, il a été considéré que les formes enthésitiques étaient des formes périphériques sans arthrite.

Les patients présentant une forme mixte ont été classés en fonction de la localisation des symptômes prédominants (axiaux ou périphériques).

Les patients ayant une atteinte de la paroi thoracique antérieure avec ostéite et une pustulose palmo-plantaire ont été classés comme SAPHO (synovite, acné, pustulose, hyperostose). 


\subsection{Analyses statistiques}

Le nombre de patients nécessaire pour rechercher l'association entre la spondylarthropathie et la maladie de Verneuil a été calculé selon les hypothèses suivantes : spondylarthropathie retrouvée chez $20 \%$ des exposés et $0,3 \%$ des non exposés, risque alpha de $5 \%$ et puissance de $90 \%$. Le logiciel NQuery 7.0 (Statistical Solutions, Ltd., Cork, Ireland) a ainsi estimé le nombre de sujets à inclure à 50 patients dans chaque groupe.

Les données ont été décrites à l'aide de moyenne et d'écart type pour les variables quantitatives et d'effectif et de pourcentage pour les variables qualitatives. L'association entre spondylarthropathie et maladie de Verneuil a été étudiée par une régression logistique conditionnelle. Parmi les patients avec maladie de Verneuil, les facteurs associés à la présence d'une spondylarthropathies ont été recherchés par une analyse univariée (tests de Wilcoxon, du Chi2 ou exacts de Fisher, selon les conditions de validité). Le seuil de signification retenu pour les analyses était de 0,05 . L'ensemble des analyses statistiques a été réalisé à l'aide du logiciel SAS version 9.3 (SAS Institute Inc., Cary, NC, USA).

\section{Résultats}

\subsection{Caractéristiques des patients}

98 patients ont été inclus dans cette étude ; parmi eux 78 patients ont été inclus dans l'analyse finale. Nous avons exclu les patients n'ayant pas réalisé I'IRM et/ou les analyses sanguines. Le flow-shart de l'étude est présenté en figure 1.

Trente-neuf patients dont 12 hommes (30.8\%) ont été inclus dans chaque groupe. L'âge moyen était de $35.6 \pm 11.1$ ans pour le groupe exposé et de $36.0 \pm 11.1$ ans pour le groupe témoin.

Pour les patients exposés, l'âge moyen lors du diagnostic de HS était de $29.7 \pm 11$ ans, avec un délai diagnostique estimé à $6.1 \pm 6.6$ années par rapport aux premiers symptômes. Six d'entre eux (15.4 \%) présentaient un antécédent familial de HS.

Concernant le grade de Hurley: 6 (15.4\%) patients étaient de grade I, $27(69.2 \%)$ de grade II et $6(15.4 \%)$ de grade III.

Trente-sept (94.9\%) patients du groupe exposé ont reçu un traitement par antibiotique(s) à visée anti-inflammatoire, avec un nombre moyen de $2.7 \pm 1.3$ 
antibiothérapies [1-6]. Une prise en charge chirurgicale a été réalisée chez 28 $(71.8 \%)$ patients avec une moyenne de 5.5 \pm 7.7 interventions. Parmi eux, 18/28 $(64.3 \%)$ ont subi une intervention large, avec une moyenne de $2.6 \pm 1.9$ interventions larges.

Vingt-deux patients (56.4\%) ont reçu une ou plusieurs biothérapies pour l'indication HS : 5 étaient sous adalimumab, 8 sous infliximab.

Parmi les 78 patients inclus dans l'étude, 5 avaient une SpA diagnostiquée avant inclusion : 4 dans le groupe HS et 1 dans le groupe témoin.

\subsection{Comparaison des données patients exposés / non exposés}

Les données des explorations cliniques, biologiques ainsi que les résultats IRM des patients exposés et non exposés sont détaillées dans le tableau 1.

\subsection{Association entre HS et SpA}

Dans le groupe exposés, 11 patients (28.2\%) avaient une SpA versus 1 (2.6\%) dans le groupe non-exposés (OR $11.0 ; 95 \% \mathrm{Cl}$ [1.4-83.3], p=0.02). Six patients avaient une SpA avec atteinte axiale isolée, 1 patient avait une atteinte périphérique (oligo arthrite), 4 présentaient une forme enthésitique (dactylite et/ou enthésite). Parmi ces derniers, 5 patients présentaient des formes combinées ( 3 formes axiales et enthésitiques, 2 formes axiales et oligoarthrites) Nous n'avons pas retrouvé de SAPHO. Le délai moyen entre l'apparition des symptômes dermatologiques et articulaires est de $46.4 \pm 67$ mois. HS a toujours précédé l'apparition du rhumatisme.

\subsection{Facteurs associés à la SpA et comparaison entre les anciens et} nouveaux cas de SpA diagnostiqués parmi les patients HS.

La présence de l'antigène HLA B27 est associée significativement au développement d'une SpA chez les patients présentant une HS. On note une tendance pour les patients ayant un antécédent personnel de psoriasis et/ou de maladie inflammatoire chronique intestinale $(p=0.06)$ ou en présence d'un taux élevé de CRP $(23.5 \pm 38.6, p=0.051)$. Le tableau 2 présente la comparaison entre les patients avec une HS ayant une SpA et ceux n'en ayant pas. 
La comparaison entre les patients ayant une SpA connue et nouvellement diagnostiquée est résumée dans le tableau 3. Bien que le nombre de patients dans chaque groupe soit faible, dans le groupe où la $\mathrm{SpA}$ était connue antérieurement, $100 \%$ des patients étaient HLA B27 positifs.

\section{Discussion}

A notre connaissance, ce travail représente la première étude exposés / non exposés s'intéressant à la relation entre HS et SpA. Nous avions pour objectif de mettre en évidence un risque accru de développer une spondyloarthrite chez les patients présentant une maladie de Verneuil ; nous avons pu le démontrer avec un OR à 11.0 .

On note une prévalence de $28.2 \%$ de SpA dans le groupe de patients atteints de HS alors qu'elle n'est que de 0.4 à $1 \%$ dans la population générale [5,6]. L'étude de Richette et al. [14], portant sur 640 patients ayant une HS rapporte une prévalence de $3.7 \%$ en utilisant les critères ESSG de SpA. Cette différence de prévalence s'explique en partie, par les différences méthodologiques pour porter le diagnostic de SpA.

Notre étude utilise le design cas/contrôle et tous les sujets inclus ont bénéficié d'un examen clinique, d'une recherche du HLA B27, d'un dosage de la CRP et d'une IRM des sacro-iliaques. Dans l'étude de Richette et al, un tiers des patients ont eu une IRM des sacro-iliaques et une recherche de l'antigène HLA B27.

Néanmoins certaines limites de notre travail méritent d'être soulevées. Tout d'abord, nous avons utilisé uniquement les critères ASAS pour porter le diagnostic de SpA. Nous n'avons pas réalisé de radiographies ou de tomodensitométrie, raison pour laquelle les critères d'Amor et de l'ESSG n'ont pu être utilisés, à l'inverse du travail de Richette et al. Ensuite, un nombre relativement élevé de patients n'ont pas réalisé d'IRM des sacro iliaques, menant à l'exclusion de 9 patients dans le groupe HS. Enfin, nous ne pouvons pas exclure la possibilité d'un biais de sélection : en effet, les patients avec une HS et des manifestations rhumatologiques ont certainement accepté plus volontiers de participer à l'étude. 
Contrairement aux résultats objectivés par Richette et al. [14] nous n'avons pas mis en évidence de lien entre la sévérité de l'atteinte cutanée ou de la qualité de vie et l'existence d'une SpA. Le grade 2 de Hurley est le plus représenté : un parallèle pourrait être établi avec le rhumatisme psoriasique où la sévérité des lésions n'est pas corrélée avec l'activité de l'atteinte articulaire, comme le rapporte Thaci et al. [21].

Les SpA retrouvées sont principalement de forme axiale, mais peuvent également être combinées avec une atteinte périphérique articulaire [22,23]. L'atteinte périphérique la plus fréquente est une mono ou oligoarhrite asymétrique, touchant préférentiellement les membres inférieurs [13] [14]. Sept nouveaux patients ont donc été diagnostiqués et comme indiqué dans le tableau 3 ils présentaient une forme pauci symptomatique avec des dactylites ou enthésites ; cela pourrait expliquer le retard diagnostique chez ces patients.

Par ailleurs, le traitement pouvant être proposé va être impacté par la présence de l' HS. En effet, parmi les différentes lignes thérapeutiques utilisées dans I' HS en fonction du grade de Hurley, les AINS peuvent être employés [24] Néanmoins cette pathologie reste associée à un surrisque significatif cardio-vasculaire[25] Or les AINS représentent le traitement de première intention des $S p A$, qu'elles soient axiales ou périphériques [26] bien que les AINS soient également connus pour augmenter le risque cardio vasculaire. Ainsi le rapport bénéfice/risque doit être soigneusement évalué dans le choix de la stratégie thérapeutique pour l'HS. De plus, la récurrence de la maladie los de l'utilisation discontinue des corticostéroïdes peut arriver [27], et le même phénomène peut être observé avec les AINS.

Concernant l'analyse IRM, nous n'avons pas trouvé de différence sur la prévalence de lésions inflammatoires entre les deux groupes. Cela s'explique par la forte proportion de patients sous anti TNF alpha (56\%) dans le groupe HS lors de la réalisation de l'IRM, il est démontré que ces traitements diminuent et peuvent faire disparaitre les signaux inflammatoires en IRM $[28,29]$. Dans notre étude, les lésions structurales en IRM n'ont été mises en évidence que dans le groupe SpA.

Étant donné la corrélation modérée dans l'interprétation des radiographies de sacroiliaque [30] nous avions choisi de retenir le diagnostic de sacro-iliite structurale 
par l'IRM, selon les données de l'étude récente de Weber [20] , qui montre que la présence d'au moins 2 érosions ou une ankylose est associée avec une bonne sensibilité au diagnostic de SpA sans perdre en spécificité.

\section{Conclusion}

Nos résultats confortent donc les données de la seule étude prospective menée à bien à ce jour ; ils s'appuient sur des arguments forts, avec la recherche de critères ASAS et notamment la réalisation systématique d'une IRM à l'ensemble des patients inclus dans l'étude. II existe une association en SpA et HS et l'atteinte axiale le plus souvent retrouvées. Un interrogatoire minutieux à la recherche de symptômes articulaires doit donc être mené chez les patients souffrant de cette affection dermatologique afin de ne pas augmenter l'errance diagnostique et permettre une meilleure prise en charge de ces patients.

\section{Financement}

Cette étude a été financée par «Appel à projets Jeunes Chercheurs - APJC 2012 du GIRCI Est », l'hôpital de Reims était le sponsor de l'étude.

\section{Pas de conflit d'intérêt}




\section{Références}

1 Acquacalda E, Roux CH, Albert C, Breuil V, Passeron T, Euller-Ziegler L. New onset of articular inflammatory manifestations in patients with hidradenitis suppurativa under treatment with infliximab. Jt Bone Spine Rev Rhum Published Online First: 13 March 2015.

doi:10.1016/j.jbspin.2015.01.020

2 Jansen I, Altmeyer P, Piewig G. Acne inversa (alias hidradenitis suppurativa). J Eur Acad Dermatol Venereol JEADV 2001; 15:532-540.

3 Matusiak L, Bieniek A, Szepietowski JC. Psychophysical aspects of hidradenitis suppurativa. Acta Derm Venereol 2010; 90:264-268.

4 Abbara S, Georgin-Lavialle S, Stankovic Stojanovic K, Bachmeyer C, Senet P, Buob D, et al. Association of hidradenitis suppurativa and familial Mediterranean fever: A case series of 6 patients. Jt Bone Spine Rev Rhum Published Online First: 26 May 2016.

doi:10.1016/j.jbspin.2016.02.021

5 Jemec GB, Heidenheim M, Nielsen NH. The prevalence of hidradenitis suppurativa and its potential precursor lesions. J Am Acad Dermatol 1996; 35:191-194.

6 Revuz JE, Canoui-Poitrine F, Wolkenstein P, Viallette C, Gabison G, Pouget F, et al. Prevalence and factors associated with hidradenitis suppurativa: results from two case-control studies. J Am Acad Dermatol 2008; 59:596-601.

7 Saunte DM, Boer J, Stratigos A, Szepietowski JC, Hamzavi I, Kim KH, et al. Diagnostic delay in hidradenitis suppurativa is a global problem. Br J Dermatol 2015; 173:1546-1549.

8 Guet-Revillet $\mathrm{H}$, Coignard-Biehler H, Jais J-P, Quesne G, Frapy E, Poirée S, et al. Bacterial pathogens associated with hidradenitis suppurativa, France. Emerg Infect Dis 2014; 20:19901998.

9 Matusiak L, Bieniek A, Szepietowski JC. Increased serum tumour necrosis factor-alpha in hidradenitis suppurativa patients: is there a basis for treatment with anti-tumour necrosis factor-alpha agents? Acta Derm Venereol 2009; 89:601-603.

10 van der Zee HH, de Ruiter L, van den Broecke DG, Dik WA, Laman JD, Prens EP. Elevated levels of tumour necrosis factor (TNF)- $\alpha$, interleukin (IL)-1 $\beta$ and IL-10 in hidradenitis suppurativa skin: a rationale for targeting TNF- $\alpha$ and IL-1 $\beta$. Br J Dermatol 2011; 164:1292-1298.

11 Bhalla R, Sequeira W. Arthritis associated with hidradenitis suppurativa. Ann Rheum Dis 1994; 53:64-66.

12 Leybishkis B, Fasseas P, Ryan KF, Roy R. Hidradenitis suppurativa and acne conglobata associated with spondyloarthropathy. Am J Med Sci 2001; 321:195-197.

13 Rosner IA, Burg CG, Wisnieski JJ, Schacter BZ, Richter DE. The clinical spectrum of the arthropathy associated with hidradenitis suppurativa and acne conglobata. J Rheumatol 1993; 20:684-687. 
14 Richette P, Molto A, Viguier M, Dawidowicz K, Hayem G, Nassif A, et al. Hidradenitis suppurativa associated with spondyloarthritis -- results from a multicenter national prospective study. $J$ Rheumatol 2014; 41:490-494.

15 Revuz J. [Clinical evaluation of the severity of hidradenitis suppurativa]. Ann Dermatol Venereol Published Online First: 11 September 2015. doi:10.1016/j.annder.2015.07.012

16 Dufour DN, Emtestam L, Jemec GB. Hidradenitis suppurativa: a common and burdensome, yet under-recognised, inflammatory skin disease. Postgrad Med J 2014; 90:216-221; quiz 220.

17 Dougados M, Baeten D. Spondyloarthritis. Lancet Lond Eng/ 2011; 377:2127-2137.

18 Sieper J, Rudwaleit M, Baraliakos X, Brandt J, Braun J, Burgos-Vargas R, et al. The Assessment of SpondyloArthritis international Society (ASAS) handbook: a guide to assess spondyloarthritis. Ann Rheum Dis 2009; 68 Suppl 2:ii1-44.

19 Rudwaleit M, Jurik AG, Hermann K-GA, Landewé R, van der Heijde D, Baraliakos X, et al. Defining active sacroiliitis on magnetic resonance imaging (MRI) for classification of axial spondyloarthritis: a consensual approach by the ASAS/OMERACT MRI group. Ann Rheum Dis 2009; 68:1520-1527.

20 Weber U, Østergaard M, Lambert RGW, Pedersen SJ, Chan SM, Zubler V, et al. Candidate lesionbased criteria for defining a positive sacroiliac joint MRI in two cohorts of patients with axial spondyloarthritis. Ann Rheum Dis 2015; 74:1976-1982.

21 Thaçi D, Behrens F, Greger G, Burkhardt H, Gnann H, Schopf R, et al. Association between skin and joint involvement in patients with psoriatic arthritis treated with adalimumab: analysis of data from a German non-interventional study. Dermatol Basel Switz 2015; 230:213-221.

22 Fimmel S, Zouboulis CC. Comorbidities of hidradenitis suppurativa (acne inversa). Dermatoendocrinol 2010; 2:9-16.

23 Lim DT, James NM, Hassan S, Khan MA. Spondyloarthritis associated with acne conglobata, hidradenitis suppurativa and dissecting cellulitis of the scalp: a review with illustrative cases. Curr Rheumatol Rep 2013; 15:346.

24 Zouboulis CC, Desai N, Emtestam L, Hunger RE, loannides D, Juhász I, et al. European S1 guideline for the treatment of hidradenitis suppurativa/acne inversa. J Eur Acad Dermatol Venereol JEADV 2015; 29:619-644.

25 Egeberg A, Gislason GH, Hansen PR. Risk of Major Adverse Cardiovascular Events and All-Cause Mortality in Patients With Hidradenitis Suppurativa. JAMA Dermatol Published Online First: 17 February 2016. doi:10.1001/jamadermatol.2015.6264

26 Zochling J, van der Heijde D, Burgos-Vargas R, Collantes E, Davis JC, Dijkmans B, et al. ASAS/EULAR recommendations for the management of ankylosing spondylitis. Ann Rheum Dis 2006; 65:442-452.

27 Napolitano M, Megna M, Timoshchuk EA, Patruno C, Balato N, Fabbrocini G, et al. Hidradenitis suppurativa: from pathogenesis to diagnosis and treatment. Clin Cosmet Investig Dermatol 2017; 10:105-115. 
28 Lambert RGW, Salonen D, Rahman P, Inman RD, Wong RL, Einstein SG, et al. Adalimumab significantly reduces both spinal and sacroiliac joint inflammation in patients with ankylosing spondylitis: a multicenter, randomized, double-blind, placebo-controlled study. Arthritis Rheum 2007; 56:4005-4014.

29 Braun J, Landewé R, Hermann K-GA, Han J, Yan S, Williamson P, et al. Major reduction in spinal inflammation in patients with ankylosing spondylitis after treatment with infliximab: results of a multicenter, randomized, double-blind, placebo-controlled magnetic resonance imaging study. Arthritis Rheum 2006; 54:1646-1652.

30 van den Berg R, Lenczner G, Feydy A, van der Heijde D, Reijnierse M, Saraux A, et al. Agreement between clinical practice and trained central reading in reading of sacroiliac joints on plain pelvic radiographs. Results from the DESIR cohort. Arthritis Rheumatol Hoboken NJ 2014; 66:24032411. 
Figure 1: Flowchart de l' étude

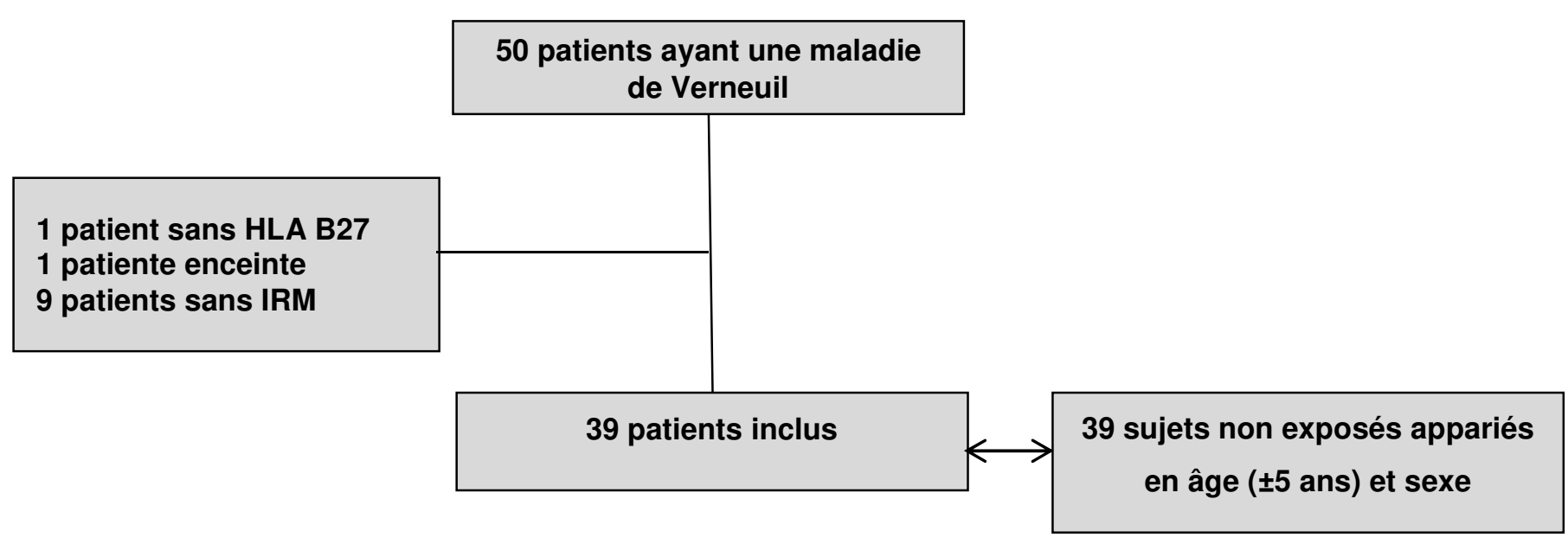


Table 1: Comparaison des patients exposés et non exposés ; résultats biologiques, analyse IRM et diagnostic de spondyloarthrite

\begin{tabular}{lcc} 
& $\begin{array}{c}\text { Exposés } \\
(\mathrm{n}=39)\end{array}$ & $\begin{array}{c}\text { Non exposés } \\
(\mathrm{n}=39)\end{array}$ \\
\hline Age (années) & $35.6 \pm 11.1$ & $36.0 \pm 11.1$ \\
Sexe masculin & $12(30.8 \%)$ & $12(30.8 \%)$ \\
Lombalgies inflammatoires & $10(25.6 \%)$ & $2(5.1 \%)^{*}$ \\
Dactylites & $8(20.5 \%)$ & $0^{*}$ \\
Antécédents d'arthrites & $11(28.2 \%)$ & $1(2.6 \%)^{*}$ \\
Uvéites & 0 & 0 \\
Antécédents de psoriasis/ MICl & $12(30.8 \%)$ & $4(10.3 \%)^{*}$ \\
CRP (mg/L) & $10 \pm 22$ & $3 \pm 7^{*}$ \\
HLA B27 & $6(15.4 \%)$ & $5(12.8 \%)$ \\
Sacroiliite inflammatoire & $8(20.5 \%)$ & $6(15.4 \%)$ \\
Sacroiliite structurale & $6(15.4 \%)$ & $0 *$ \\
SpA & $11(28.2 \%)$ & $1(2.6 \%)^{*}$
\end{tabular}

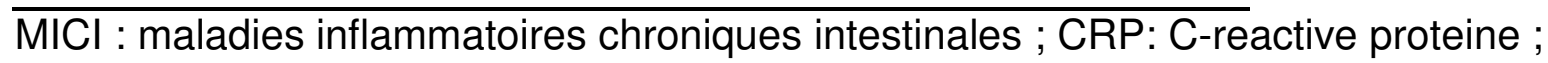
$\mathrm{SpA}$ : spondyloarthrites

${ }^{*} p<0,05$ 
Table 2: Comparaison des patients exposés, avec ou sans spondyloarthrite $H S$ Spondyloarthrite Non Spondyloarthrite $(n=11) \quad(\mathrm{n}=28)$

Sexe masculin $5(45.4 \%)$ $7(25 \%)$

Age à l'inclusion (années) $\quad 34.2 \pm 12.4$ $36.1 \pm 10.7$

Grade de Hurley

1

2

3

Durée d'évolution de l'HS

$8.9 \pm 4.7$

$12.7 \pm 10.1$

Antécédents de psoriasis /

$6(54.5 \%)$

$\mathrm{MICl}$

DLQI

$7.3 \pm 7.9$

$9.7 \pm 6.5$

Traitement par anti TNF

$7(63.6 \%)$

$15(53.6 \%)$

HLA B27 positif

$5(45.4 \%)$

$23.5 \pm 38.6$

$4(14.3 \%)$

$20(71.4 \%)$

$4(14.3 \%)$

$6(21.4 \%)$

CRP (mg/L)
$1(3.6 \%)$

$5.6 \pm 7$

HS : hidrosadénite suppurative; $\mathrm{MICl}$ : maladies inflammatoires chroniques intestinales ; DLQI : Dermatology Life Quality Index; TNF : tumor necrosis factor ; CRP : C-Reactive Proteine 
Table 3: Comparaison parmi les patients exposés des cas de spondyloarthrites connues versus nouvellement diagnostiquées.

$$
\begin{aligned}
& \text { SpA connues SpA diagnostiquées au } \\
& (n=4) \quad \begin{array}{c}
\text { cours de l'étude } \\
(n=7)
\end{array}
\end{aligned}
$$

$\begin{array}{llll}\text { Age (années) } & 33 \pm 9 & 35 \pm 15 & 0.78 \\ \text { Antécédent de psoriasis / MICI } & 2(50 \%) & 5(71.4 \%) & 0.57 \\ \text { Dactylites/ Enthésites } & 3(75 \%) & 4(57.1 \%) & 0.99 \\ \text { Antécédent d'arthrites } & 2(50 \%) & 2(28.6 \%) & 0.57 \\ \text { HLA B27 positif } & 4(100 \%) & 1(14.3 \%) & 0.01 \\ \text { CRP > 5mg/l } & 3(75 \%) & 4(57.1 \%) & 0.99 \\ \text { Sacroiliite à l'IRM } & 4(100 \%) & 5(71.4 \%) & 0.49\end{array}$

SpA : spondyloarthrite; $\mathrm{MICl}$ : maladie inflammatoire chronique intestinale ; CRP: Creactive proteine; IRM: Imagerie par resonance magnétique 